Dans cet article on analyse les traces intertextuelles qui résistent ARTICULOS · 221-231 souvent au traducteur et révèlent l'état du dialogue interculturel. L'objectif de cette étude est d'examiner les tendances générales de la traduction de l'intertextualité du lituanien en français et de cibler les problèmes du transfert culturel des traces intertextuelles dans les traductions littéraires durant la période de 1990 à 2016. En nous situant dans le domaine de la traduction de l'intertextualité, nous nous appuyons sur la méthodologie de la théorie interprétative qui sous-tend la vision de la traduction comme transfert culturel. L'analyse traductologique nous permet d'établir quatre tendances principales de la traduction de l'intertextualité. Parmi ces tendances, on pourrait noter celle de la transmission de l'implicite culturel sans y apporter de changements, celle de l'explicitation et celles de la décontextualisation et de l'omission des traces intertextuelles.

MOTS CLÉs: traduction, intertextualité, décontextualisation, théorie interprétative de la traduction.

\title{
Transfert culturel de l'intertextualité dans les traductions littéraires du lituanien en français
}

AURELIJA LEONAVIČIENĖ

Université de Vytautas Magnus
Cultural Transfer of Intertextual Units in Translations of Literary Texts from Lithuanian into French

This article analyses intertextual units which are problematic issue to the translator and which reveal the state of intercultural dialogue. The aim of the present research is to analyse the general tendencies of the translation of intertextual units from Lithuanian into French and to determine the problems of the cultural transfer of intertextual units in the translations of literary texts during the period from 1990 to 2016. In this research, the author relies on the methodology of interpretative theory of translation that treats translation as a cultural transfer.

The analysis of a series of translated texts allows us to establish four main trends in the translation of intertextual units, such as transmission of implicit meaning without changes, explicit transmission, decontextualization of the intertextual unit, and omission of the intertextual unit.

KEY WORDS: translation, culture-specific intertextual units, decontextualization, interpretative theory of translation. 


\section{INTRODUCTION}

Dans la traductologie d'aujourd'hui, on considère le texte traduit comme un ensemble sémantique complexe qui s'inscrit dans des contextes linguistiques, historiques et politiques de la culture d'arrivée. Toute traduction n'est jamais isolée; elle est en rapport avec d'autres textes, d'autres discours qui sont intimement liés à l'expérience culturelle d'un certain pays, à l'histoire d'un peuple, à une vision et une perception du monde. Chaque traducteur cherche une clé dont dispose sa propre culture pour accéder au texte littéraire de l'Autre.

Cet article a pour but d'analyser les tendances générales de la traduction de l'intertextualité du lituanien en français et de cibler les problèmes du transfert culturel des traces intertextuelles dans les traductions littéraires durant la période de 1990 à 20I6. L'intertextualité qui résiste souvent au traducteur et révèle l'état du dialogue interculturel, a été choisie comme objet d'étude sur la base d'une position formulée de longue date en traductologie, à savoir que les principaux problèmes rencontrés dans le processus de traduction proviennent non des unités linguistiques du texte ou des différences dans les sens linguistiques, mais du passage d'un système culturel à un autre.

Le phénomène de l'intertextualité est analysé dans les œuvres littéraires lituaniennes (de R. Granauskas, J. Savickis, V. Juknaite, S. Parulskis, J. Ivanauskaite, A. Landsbergis, I. Meras, S. T. Kondrotas, etc.) dont le volume excède rooo pages, et dans leurs traductions en français, de volume comparable. Les textes littéraires analysés ont été traduits du lituanien par des traducteurs de langue maternelle française. Ces traductions ont été publiées en France entre 1990 et 2016 , dans différents ouvrages, par exemple, dans l'anthologie de nouvelles lituaniennes Des âmes dans le brouillard, éditée en 2003, dans le recueil Littératures des pays baltes de 1992, dans plusieurs numéros des Cabiers Lituaniens publiés entre 2000 et 2013 , dans le recueil de poésie Poésie 92. Revue bimestrielle de la poésie d'aujourd'bui de I992, et dans diverses publications isolées telles que L'ombre du serpent de S.T. Kondrotas paru en 2007 ou Ma vie nocturne de J. Mekas paru en 2007. Afin d'éviter des distorsions méthodologiques, les textes littéraires lituaniens traduits en français non à partir de l'original lituanien, mais par l'intermédiaire de la traduction russe ont été écartés du corpus. Cela concerne notamment La saga de Juzas de J. Baltušis, Sur quoi repose le monde de I. Meras ou La forêt des Dieux de B. Sruoga.

\section{INTERTEXTUALITÉ COMME LE PROBLÈME THÉORIQUE DE LA TRADUCTION}

En nous situant dans le domaine de la traduction de l'intertextualité, nous nous appuyons sur la théorie interprétative qui sous-tend la vision de la traduction comme transfert culturel. Dans cette optique, la traduction est considérée comme un dialogue entre deux cultures et l'objet à traduire, tel qu'il est compris actuellement, est envisagé comme le vouloir-dire ou " le produit de tout un réseau de relation extra-, inter- et intra- textuelles dont il tire sa pleine substance " (Israël, 2005: 64). Conformément à ce qui a été dit, aucun texte n'est isolé, « il vient après d'autres textes et certainement avant d'autres encore qui vont suivre » (Durieux, I998: 22). Un texte qui communique avec des autres textes, "se construit comme une mosaïque de citations ", comme l'indique Julia Kristeva (I969: 85). Par conséquent, les traces intertextuelles absorbées et transformées dans le texte acquièrent leur sens en s'associant aux contextes 
situationnels, culturels et aident à construire le sens global du texte.

Constatant que l'intertextualité se manifeste par des formes différentes, nous nous appuyons sur les recherches de Geneviève Roux-Faucard, professeur de l'Université de Paris III (ESIT), qui décrit quatre types de traces intertextuelles provenant des autres textes. Selon G. Roux-Faucard, ces traces se présentent par une citation qui est un emprunt littéral et déclaré, aussi, par une référence qui est un emprunt non littéral mais déclaré, ou encore, par une allusion qui est un emprunt non littéral et non déclaré, et finalement, par un emprunt littéral et non déclaré (2006: 102-103). « Face à la traduction, ces quatre types de traces ont des comportement relativement différents »- constate G. Roux-Faucard et apporte encore quelques précisions : " Les difficultés de la traduction et les méthodes employées pour les résoudre sont donc variables selon la forme de la trace » (2006: 104).

Il est évident que la traduction n'est pas une pratique linéaire qui s'inscrit entre le texte original et le texte traduit. C'est un processus cognitif, ouvert à l'interprétation. "Quel que soit le paradigme théorique adopté, le traducteur ne dispose certes matériellement que du texte à traduire sous forme de graphismes imprimés sur un papier, mais au lieu d'en rester à l'expression brute, il va chercher au-delà des mots et des phrases - du dire - pour en extraire le vouloir-dire et ainsi accéder au sens ; c'est le sens ainsi appréhendé qu'il va réexprimer dans l'autre langue. » - précise Christine Durieux (1998: 19). Conformément au parcours proposé, les traductologues lituaniens actuels (Ragaišienè, 2008: 44-50 ; Leonavičienè, 2013: 97-IO8 ; 20I4: 45-64; etc.) analysent les problèmes de la traduction de l'intertextualité dans le contexte du sens global et dans l'ensemble holistique de l'œuvre. Dans les études traductologiques, les chercheurs se posent souvent des questions suivantes : que faudrait-il faire pour surmonter les difficultés d'ordre culturel ? Comment transmettre le sens culturel des traces intertextuelles?

Comme nous le savons déjà, la traduction de l'intertextualité s'inscrit dans la représentation de l'implicite de la culture de l'Autre qui s'articule sur de multiples niveaux. Le problème de l'implicite, essentiel en ce qui concerne la problèmatique de la traduction de la culture, vient de la capacité du traducteur de saisir les manifestations de la culture étrangère de l'Autre et de savoir comment référer à un monde qui n'est pas le sien. À ces observations il convient d'ajouter la notion de " compétence intertextuelle » du traducteur et du lecteur réel, « celle-ci étant définie comme la connaissance, antérieure à la lecture du texte citant, des textes cités et des moyens habituels d'y faire référence " (Roux-Faucard, 2006: I06). Dans bien des cas, les traducteurs se posent une question : soit aller à la rencontre de la culture de l'Autre, soit assurer une grande lisibilité par l'adaptation qui pourrait conduire à la destruction culturelle du texte, à son annexion. L'idée de l'annexion dans la traduction, la pratique ethnocentrique ainsi que le danger de l'installation d'un lecteur de la traduction sur un piédestal, sont bien exploités chez Jean-Louis Cordonnier qui présente l'ethnocentrisme comme le rejet de la culture de l'Autre « en soumettant au Même le visage de l'Autre » (1995: 167-186).

Chaque fois qu'on parle de la traduction de l'intertextualité, on se souvient que le vécu socioculturel n'est pas identique d'une communauté à l'autre et qu'une bonne maîtrise de l'implicite de l'Autre demande de l'expérience des contacts culturels répétés. Dans le cas de manque de ces contacts et de connaissances

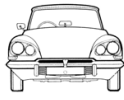

223 


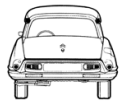

224 culturelles du traducteur, les «traductions initiales sont celles où l'on constate le plus de pertes » (Roux-Faucard, 2006: 108). Vu sous cet angle, on pourrait se poser une question : comment améliorer la qualité de la traduction de l'implicite et comment rapprocher les cultures à travers la traduction ? La réponse est donnée par Marianne Lederer qui dit qu'il faut « une multitude de textes traduits pour que se crée progressivement une image qui parvienne à dissiper l'ignorance et à rapprocher les civilisations » (I994: I28).

\section{TRADUCTION DE L'INTERTEXTUALITÉ DANS LES GEUVRES LITTÉRAIRES DU LITUANIEN EN FRANÇAIS}

En analysant le matériau bilingue, l'intertextualité des textes de la littérature lituanienne est étudiée comme un composant d'un ensemble holistique, dans le contexte de la signification globale de l'œuvre, car, comme l'affirme HansGeorg Gadamer, les parties, qui sont définies par l'ensemble, définissent à leur tour l'ensemble (1999: 27). Sur la base de ce qui a été dit, notre étude soutient des principes fondamentaux de la théorie interprétative de traduction et le point de vue du philosophe Paul Ricœur, selon lequel la tâche du traducteur n'est pas orientée du mot à la phrase, au texte ou à la réalité culturelle, mais dans la direction opposée : en se chargeant des idées innombrables et des interprétations d'une culture concrète, le traducteur descend du texte à la phrase et au mot (20I0: $4 \mathrm{I}$ ).

Après avoir fait l'analyse des textes littéraires lituaniens, dont le volume est plus de 1000 pages, nous avons trouvé 253 traces intertextuelles parmi lesquelles nous avons constaté I4I allusions qui sont des emprunts non littéraux et non déclarés, 49 emprunts littéraux et non déclarés, c'est-à-dire, des citations non déclarées, 35 citations qui représentent un emprunt littéral et déclaré, et 28 références qui sont considérées commes des emprunts non littéraux mais déclarés (voir tableau I). Cette classification est basée sur la classification proposée par G. Roux-Faucard. Toutes ces traces intertextuelles sont reconnues comme provenant des autres textes ou discours. Les résultats de l'analyse quantitative nous permetent de constater la prédominance des allusions et des emprunts littéraux, non déclarés par rapport aux citations et aux références dans les ouvrages de la littérature lituanienne.

Toutes ces traces de l'intertextualité permettent aux textes littéraires de produire " un maximum de sens avec un minimum de signifiant » - comme le dit G. Roux-Faucard dans "Intertextualité et traduction " (2006: I05). Constatant que l'intertextualité aide à construire le sens global du texte, nous nous posons les questions suivantes : est-ce que le

Tableau I

Manifestation des traces intertextuelles dans les œuvres analysées

\begin{tabular}{llcc}
\hline No & Manifestations de l'intertextualité & Nombre d'exemples & \% d'exemples trouvés \\
\hline I. & Allusion qui est un emprunt non littéral et non déclaré & I4I & $56 \%$ \\
2. & Emprunt littéral et non déclaré & 49 & I9\% \\
3. & Citation qui est un emprunt littéral et déclaré & 35 & I4\% \\
4. & Référence qui est un emprunt non littéral mais déclaré & 28 & II\% \\
\hline & Total & 253 & Io0\%
\end{tabular}


traducteur français arrive toujours à identifier le sens intertextuel dans le contexte de la signification globale de l'œuvre lituanien? Est-ce qu'il arrive à surmonter les difficultés d'ordre culturel dans la traduction de l'intertextualité ? Pour répondre à ces questions, nous allons examiner comment les problèmes posés par l'intertextualité sont-ils résolus dans les traductions françaises.

\section{Transmission de l'implicite culturel sans y apporter de changements}

Les résultats de l'analyse traductionnelle de l'intertextualité permetent de constater la prédominance de la tendance de transmission de l'implicite culturel, c'est-à-dire, de la présence culturelle du texte original dans le texte traduit sans y apporter de changements. C'est le cas de connaissances culturelles proches quand l'intertextualité est familière aux lecteurs des cultures lituanienne et française. Les exemples de ce type constituent $5 \mathrm{I} \%$ de renvois intertextuels qui représentent des références communes littéraires évoquées par l'intermédiaire du nom des personnages, des allusions ou des emprunts à la Bible. Pour le montrer, nous présentons quelques exemples :

Visai ne mano pelenè, toji skaisčioji princesè. Aš èmiau sirgti batų liga, kai pagalvodavau, kur tie mistiški batai. Kaip detektyvas koks. Pelenę reikia surasti. (Savickis, 2006: 60)

Ce n'est vraiment pas ma Cendrillon, cette princesse lumineuse.

Je commençais à souffrir de la maladie des chaussures, et je réfléchissais à l'endroit où ces mystérieuses chaussures pouvaient bien se cacher. Je suis une sorte de détective, et il me faut trouver cette Cendrillon. (Savickis, 2003: 254)
Šių namų šeimininkè — įsikūnijęs kunigaikštis Drakula. Dabar jis žiūri televizorių, tačiau naktį, brangieji, nakti... (Ivanauskaitė, 2003a: r68)

La maîtresse de la maison est la réincarnation de Dracula. Maintenant elle regarde la télévision, mais cette nuit, mes chers amis, cette nuit... (Ivanauskaitè, 2003b: I65)

Jos dèka jis išgyveno prieš trisdešimt su kaupu metų, išgyvens ir dabar, nes jo Penelopẻ laukia atstūmusi jaunikius, laukia sugrịžtančio nuo plačios, niūrios upès krantų. (Gavelis, I989: 49)

Grâce à elle, il avait survécu plus de trente ans, et il survivrait encore, car sa Pénélope l'attendait ; repoussant tous ses prétendants, elle attendait qu'il revienne des bords de la grande rivière sombre. (Gavelis, 2003: 283)

Mamutè tebesimeldè.

Viešpaties Angelas apreiškè Marijai: Tu pradèsi iš Šventosios Dvasios. Sveika, Marija, malonès pilnoji! Viešpats su tavimi...

Čia Morta nutilo, galvą nuleido. (Meras, I995: 71)

Sa petite mère continuait de prier.

-L'Ange de Seigneur est apparu à Marie : tu enfanteras par le Saint-Esprit. Salut Marie pleine de grâce. Le Seigneur est avec toi...

Ici, Morta se tut, baissa la tête. (Meras, 2003: 92-93)

En nous fondant sur les premiers trois exemples cités, nous voyons que la perception de l'intertextualité suscite une image pareille dans les deux cultures, c'est-à-dire, les lecteurs français et lituaniens sont familiers à l'histoire de Cendrillon (en lituanien - Pelenè) de Charles Perrault ou au personnage Dracula de Bram Stoker, écrivain et romancier irlandais, ou encore à Pénélope, le personnage de l'Odyssée d'Homère. Ces références culturelles permettent d'identifier l'intertextualité et la rendre présente à l'esprit du lecteur de la culture d'accueil.

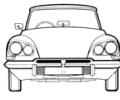

225 
En ce qui concerne le quatrième exemple, il présente un emprunt à la Bible qui est considérée comme familière pour les cultures européennes. Malgré les nuances de formulation ou des versions de ce texte sacré, les lecteurs lituaniens et français ont une compétence nécesssaire pour identifier des traces intertextuelles empruntées à la Bible. C'est la raison pour laquelle le traducteur choisit la transmission de l'intertextualité par une simple correspondance.

\section{Explicitation}

Pour aller plus loin, nous nous intéressons à l'explicitation qui aide au traducteur de modifier « le rapport implicite / explicite de l'original pour atteindre un nouvel équilibre implicite / explicite dans sa langue »- comme nous précise Marianne Lederer (I994: I26). Notre inventaire de la traduction de l'intertextualité dans les ouvrages analysés fait apparaître $29 \%$ d'exemples dont la traduction est marquée par l'explicitation qui aide à élargir un espace sémantique du texte par l'introduction des notes en bas de page ou d'une brève explicitation dans le texte. Citons un exemple de l'explicitation par l'introduction de la note en bas de page :

Seklyčia tapo užrakinta ir kaipo šventovė, žalčių prileista, buvo saugojama ir niekas neįleidžiamas. (Savickis, I997: 42)

La chambre d'amis fut fermée à clé et, comme un sanctuaire réservé aux couleuvres, ${ }^{\mathrm{I}}$ elle fut surveillée et personne n'eut le droit d'y entrer. (Savickis, 200I: 55)

Les traces intertextuelles qui sont mises en gras dans l'exemple cité, introduisent des contextes extralinguistiques et exigent une lecture non linéaire du texte. Plongé dans cette lecture, le

\footnotetext{
I « La couleuvre était un animal sacré dans la Lituanie païenne » (N.D.T.).
}

traducteur fait le choix entre deux voies d'accès qui « s'offrent au problème posé par l'acte de traduire : soit prendre le terme «traduction » au sens strict de transfert d'un message verbal d'une langue dans une autre, soit le prendre au sens large, comme synonyme de l'interprétation de tout ensemble signifiant à l'intérieur de la même communauté linguistique "(Ricœur, 2004: I8). Dans le cas de la traduction des textes qui contiennent des traces intertextuelles, le traducteur privilégie la deuxième voie proposée par Ricœur. Il va sans dire que dans le transfert de l'intertextualité, il s'agit non seulement de reconnaître et de comprendre le sens des traces intertextuelles, mais aussi de « donner au lecteur étranger des connaissances supplémentaires, minimum mais suffisantes pour entr'ouvrir la porte qui mène à la connaissance de l'autre » (Lederer, I994: I23).

Dans le but de restituer le vouloir dire de l'auteur du texte original, les traducteurs utilisent souvent les notes en bas de page tenant comte de ce fait que le lecteur français ne dispose d'une même compétence intertextuelle que le lecteur lituanien et semble avoir besoin d'une explicitation. C'est pour cette raison que le traducteur de l'exemple 5 apporte des informations sur le non-dit de l'Autre : il précise le rôle de la couleuvre dans la culture de la Lituanie païnne d'autrefois. La couleuvre, comme nous le dit Ugnè Karvelis, représente un « animal sacré du panthéon païen, paisible gardien des foyers, choyé par les hommes qui lui offrent du lait » (I998: 5). Pour montrer l'importance de la couleuvre dans la culture lituanienne paienne, nous nous référons à la Mythologie générale Larousse I935 qui présente plus d'informations : « D'après des témoignages très sûrs (ceux de Praetorius et de Bretkunas), chaque Lituanien gardait, à l'époque du paganisme, une couleuvre dans sa maison. On s'adressait à des sorciers spéciaux 
qui introduisaient la couleuvre dans la demeure, où un lit lui était réservé dans un coin; les habitants la soignaient et nourrissaient avec un amour et un respect religieux, car la couleuvre était considérée comme la protectrice de la maison.» (cité par Lefebvre, 2005: 26).

Introduite dans la nouvelle de Jurgis Savickis, l'allusion à la mythologie lituanienne, notamment au "sanctuaire réservé aux couleuvres ", représente une stratégie textuelle qui donne le moyen de localiser l'intertexte dans le nouveau contexte de la vie de la Lituanie du XX-ième siècle et de faire de l'ironie dans le témoignage des survivances du paganisme dans le subconscient des personnages. Face à la traduction de cette trace intertextuelle, le traducteur français reconnaît facilement le contexte caché et se porte sur une solution d'élargir un espace sémantique de l'implicite dans la note en bas de page. À notre avis, cette explicitation suscitée par la note du traducteur est suffisament informative et montre le choix du traducteur d'aller à la rencontre de la culture de l'Autre.

Pour donner au lecteur français des connaissances supplémentaires et présenter l'implicite de l'Autre, les traducteurs choisissent aussi une brève explicitation à l'intérieur du texte, par exemple :

Pats užsakydavo laikraščius kaimynams, o dažniausiai - savo mégstamiausią žurnalą „Lurdas“ ir savaitraštį „Mūsų laikraštis“. (Žindžiuvienè-Deksnytè, 200o: 584 )

Il commandait lui-même les journaux pour les villageois, et très souvent sa revue préférée Lurdas (Lourdes) et l'hebdomadaire Mūsu laikraštis (Notre journal). (Žindžiuvienè-Deksnyte, 2008: 49)

Dans le cas des périodiques à grand tirage, les traducteurs conservent le plus souvent les titres d'origine, mais en ce qui concerne les périodiques moins connus, ceux-ci deviennent l'objet d'une explicitation. Cette tendance de la traduction des traces intertextuelles permet au traducteur de transmettre le vouloir dire de l'auteur dans sa plénitude et son effet. Malgré les avantages de l'explicitation, on se demande si les suppléments informationnels sont toujours nécessaires ou encore, s'ils ne sont pas superflus.

Partant d'expériences personnelles dans le but de "dire presque la même chose ", les traducteurs se posent " la vieille question de savoir si une traduction doit conduire le lecteur à s'identifier à une certaine époque et un certain milieu culturel - celui du texte original - ou si elle doit rendre l'époque et le milieu accessibles au lecteur de la langue et de la culture d'arrivée » (Eco, 2006: 215-216). Si le traducteur conduit le lecteur à la rencontre de l'original, il est souvent obligé d'introduire des notes pour l'explicitation de l'implicite. Dans ce cas, la traduction s'entourent de plusieurs notes qui offrent le paratexte du texte traduit et risque à son tour de perdre la cohérence textuelle ou de se transformer en édition savante. En nous fondant sur les exemples trouvés dans les textes analysés de deux langues, nous n'observons pas de grande fréquence de notes qui puissent modifier les conditions de la lecture dans la culture d'accueil.

\section{Omission et décontextualisation des traces intertextuelles}

Leétude de la traduction de l'intertextualité fait apparaître $2 \%$ d'exemples trouvés dont les traces intertextuelles ont été omises dans les traductions françaises. Cette troisième tendance se justifie par une orientation plus marquée du traducteur vers le destinataire français et vers ses connaissances culturelles.

Après avoir étudié la traduction de l'implicite culturel des ouvrages littéraires lituaniens,

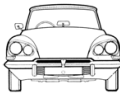

227 


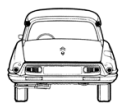

228 nous constatons la quatrième tendance de la traduction de l'intertextualité, celle de la décontextualisation à son maximum « lorsque dans la culture d'accueil, la connaissance du texte cité peut être considérée comme nulle » (Durieux, I998: I06). Les exemples qui prouvent la décontextualisation des traces intertextuelles, constituent I8 \% d'exemples trouvés. Afin d'illustrer ce phénomène, nous présentons un exemple tiré d'une nouvelle "Genèse d'une chanson » (Dainos gimimas) de Algirdas Landsbergis et de la traduction de cette nouvelle en français :

[...] žiūri kaip nekalti, tyli, o velniai žino, ką jie galvoja? vakar tas tenoras raitytais plaukais, ir per ilgais, sukišę nosis su čeke šnabždasi, net krūptelëjo mane pamatęs, aš ì knygutę įsirašiau, gal apie Kauno chuliganus pasakojo, ar tuos Prahos ir Kauno susideginusius pamišèlius lygino, supranti? (Landsbergis, I992: I82)

[...] ils regardent comme des innocents, ils se taisent, mais le diable sait ce qu'ils pensent? Hier, ce ténor aux cheveux ondulés et trop longs qui chuchotait, nez à nez avec une tchèque, il a même sursauté quand il m'a vu, je l'ai noté dans mon calepin, peut-être qu'il parlait des voyous de Kaunas, ou bien qu'il comparait ces timbrés de Prague et de Kaunas qui se sont immolés, tu comprends? (Landsbergis, 2003: I2O)

Dans cet exemple, l'auteur du texte original présente des réflexions du personnage principal qui est un agent du Comité pour la Sécurité de l'État (KGB) de l'URss. En parlant de son travail, ce personnage fait une allusion aux événements politiques et historiques de 1972 quand les jeunes de Kaunas sétaient révoltés contre le régime soviétique en Lituanie. L'écrivain fait un rapprochement intertextuel entre les événements politiques de Lituanie et le Printemps de Prague de I968, mais l'implicite de l'intertextualité échappe au traducteur. Il n'arrive pas à saisir des allusions qui se réfèrent à un monde qui n'est pas le sien, par conséquent, il reste incapable de restituer le fait socioculturel. Et le paradoxe est que le traducteur qui ne révèle pas d'implicite trouve nécessaire d'expliciter le nom propre de Kaunas dans la note en bas de page. Il écrit : "Ancienne capitale de la Lituanie, deuxième ville du pays (N.D.T.) »! On pourrait se poser une question : pourquoi le traducteur présente-t-il la ville de Kaunas, quand le lecteur français est capable de trouver toutes les informations nécessaires sur les sites Internet ou dans les encyclopédies. Quand aux événements politiques présentés dans l'exemple cité, ils restent inconnus du lecteur et du traducteur, mais celui-ci aurait dû mener une recherche documentaire afin de « donner au lecteur étranger des connaissances supplémentaires »comme le dit M. Lederer (I994: I23).

Il est évident que la pratique de traduire se réalise dans l'intertextualité, et tout texte, quel qu'il soit, et en rapport avec d'autres textes. C'est pourquoi le traducteur devrait avoir une compétence intertextuelle ainsi qu'une compétence culturelle parce que le vécu socioculturel n'est pas identique d'une culture à l'autre. Encore une fois, nous proposons un exemple qui représente une allusion littéraire et sa traduction en français :

[...] ar kas tą mamytès rateli dar pasuka? dulkèm apneštas, dulkèm, pelèsiais ir kerpèm apaugus aukštai... pilis ant kalnų, kaip Lietuvoj... (Landsbergis, 1992: I89)

[...] quelqu'un fait-il encore tourner le rouet de maman? Couvert de poussière, de poussière, de moisissures, et on coupe ce qui pousse trop haut!!! Le château dans les montagnes, comme en Lituanie!!! (Landsbergis, 2003: 130)

Dans cet extrait de la nouvelle « Genèse d'une chanson " de A. Landsbergis, le personnage 
principal se souvient de son passé lointain en Lituanie qui se caractérise par une image du château recouvert de mousse et de lichens. Par cette image, le personnage fait une allusion au poème « Le château de Trakai » de Maironis, poète le plus célèbre du romantisme lituanien. Comme la poésie de ce poète s'inscrit dans le programme de l'enseignement de littérature lituanienne des écoles scondaires, presque tous les éléves des classes terminales savent par cœur le poème «Le château de Trakai ». Présentons la première strophe de ce poème pour pouvoir comparer avec l'implicite de la nouvelle « Genèse d'une chanson » :

Pelèsiais ir kerpèm apaugus aukštai

Traku štai garbinga pilis!

Jos aukštus valdovus užmigdè kapai, $\mathrm{O}$ ji tebestovi dar vis.

Bet amžiai bèga, ir griūvančios sienos Kas dieną nyksta, apleistos ir vienos!..

(Maironis, 2012: 80)

En guidant le procesus de la traduction, le traducteur français a utilisé tout son bagage cognitif mais celui-ci était insuffisant pour saisir et transmettre une poétique de l'allusion. En faisant une traduction linéaire, le traducteur n'a pas su répérer l'implicite et par conséqent, il n'a pas pu le transmettre au nouveau lecteur de la culture d'accueil. Ce qui est évident, c'est que le traducteur s'est trompé entre le sens des mots lituaniens « kerpé » qui signifie «le lichen ", et le mot « kirpti » qui signifie « couper». Le résultat de cette traduction linéaire est la décontextualisation de la trace intertextuelle et l'apparition de la phrase « et on coupe ce qui pousse trop haut !!! » qui n'a rien à voir avec l'implicite du texte original. Si on juge par cette innovation présentée, on ne peut pas dire que c'est le rejet de l'étrangéité de l'Autre ou la pratique ethnocentrique appliquée par le tra- ducteur. C'est plutôt une manque de connaissances culturelles de l'Autre et le problème de compréhension.

Il faut noter que le poème "Le château de Trakai » a été traduit en français par Ugnè Karvelis et a été publié dans la Revue bimestrielle de la poésie d'aujourd'hui à Paris en I992. Citons la première strophe de la traduction du poème :

De mousse, de lichens recouvert, Voici le noble château de Trakai !

Ses seigneurs puissants dorment dans la tombe,

Mais lui se dresse hautain.

Hélas, les siècles passent, et ses murs lézardés Croulent, solitaires, abandonnés (85).

Nous sommes de l'avis que le traducteur aurait pu utiliser cette traduction pour transmettre les traces intertextuelles du poème de Maironis dans la nouvelle «Genèse d'une chanson » dont la traduction est paru presque dix ans plus tard, c'est-à-dire, en 2003. Bien sûr, le traducteur est libre de choisir comment construire le sens global du texte mais, selon la théorie interprétative, « il ne dispose pas de cette liberté par rapport au sens » qui est « compris non pas en tant qu'esprit par opposition à la lettre, mais en tant qu'effet global du texte sur le destinataire » - précise Marianne Lederer (I994: 86).

\section{CONCLUSIONS}

Après avoir étudié 48 traductions du lituanien en français, nous avons établi quatre tendances principales de la traduction de l'intertextualité durant les 26 dernières années. Parmi ces tendances, il faut noter celle de la transmission de l'implicite culturel sans y apporter de changements, celle de l'explicitation et celles de la décontextualisation et de lomission des traces intertextuelles.

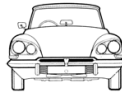

229 


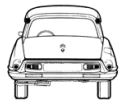

230
Face à l'analyse traductionnelle dont le volume excède 2000 pages en deux langues, nous avons pu cibler les problèmes de transfert culturel de l'intertextualité, parmi lesquels le plus important est celui de décontextualisation au maximum des traces intertextuelles.

Comme nous avons déjà constaté, l'intertextualité de la littérature lituanienne a été étudiée dans le contexte du sens globale et dans l'ensemble holistique de l'œuvre. La conséquence de cette vision est que la tâche du traducteur consistait à comprendre l'implicite de l'Autre dans le contexte du sens globale de l'œuvre et de savoir comment référer à un monde qui n'est pas le sien. Par conséquent, le traducteur ne devait pas manquer de compléments cognitifs et d'éléments extra-linguistiques qui s'ajoutent au texte pour construire le sens.

À travers l'analyse de la traduction de l'intertextualité, nous pouvons arriver à la conclusion que certains traducteurs français, malgré leur bonne maittrise de la langue lituanienne, n'avaient pas eu de connaissances culturelles suffisantes pour saisir l'implicite de l'Autre. Il faut noter que la plupart des traducteurs français ne sont pas passés par une formation spécifique de la traduction et de la culture lituanienne. La grande majorité d'entre eux ont fait des études de langues étrangères ou de lettres et ensuite, après avoir appris la langue lituanienne, ils se sont lancés à la traduction. Bien sûr, rien ne s'oppose que les principes de la traduction interprétative soient appliqués à la traduction littéraire mais les résultats de notre analyse montrent qu'il ne suffit pas d'études des langues pour passer d'une culture à l'autre.

Dans notre étude, il ne s'agit pas de règles absolues de la traduction mais de choix individuels, choix différents suivant le texte littéraire, sa situation de communication, le bagage cognitif du lecteur et du traducteur. Les résultats de l'analyse du transfer culturel de l'intertextualité permettent de constater que les traducteurs français ont conservé un certain équilibre dans leurs solutions de la traduction de l'intertextualité. Après avoir décontextualisé et omis 20 $\%$ d'exemples trouvés où l'intertextualité est visible, les traducteurs sont restés prudents pour d'autres cas de l'implicite culturel et ils ont tenté de familiariser le lecteur français à la culture lituanienne.

RECIBIDO EN JULIO DE 2017 ACEPTADO EN DICIEMBRE DE 2018 VERSIÓN FINAL DE ENERO DE 2019

\section{RÉFÉRENCES BIBLIOGRAPHIQUES}

\section{Bibliographie Primaire}

Gavelis, Ričardas (I989): «Berankis», Novelés metai I988, Vilnius: Vaga, 39-57.

Gavelis, Ričardas (2003): «Le manchot», tr. Muriel Puig, en Loreta Macianskaite (ed.) Des âmes dans le brouillard, Caen: Presses Universitaires de Caen, 265-293.

IvanAus KAItĖ, Jurga (2003a): «Pakalnučių metai», Pakalnučiu metai, Vilnius: Tyto alba.

IVANAUSKAITE், Jurga (2003b): «L'année des muguets», tr. Marielle Vitureau, en Loreta Macianskaite (ed.) Des âmes dans le brouillard, Caen: Presses Universitaires de Caen, I53-I67.

Kondrotas, Saulius Tomas (I98I): Žalčio žvilgsnis, Vilnius: Vaga.

Kondrotas, Saulius Tomas (2003): L'ombre du serpent, tr. Ugnė Karvelis, Paris: Éditions Albin Michel S. A.

Landsbergis, Algirdas (I992): «Dainos gimimas», Keliones muzika, Vilnius: Vaga, I80-190.

Landsbergis, Algirdas (2003): «Genèse d'une chanson», tr. Muriel Puig, en Loreta Macianskaite (ed.) Des âmes dans le brouillard, Caen: Presses Universitaires de Caen, II5-I3I.

Maironis (20I2): "Traku pilis», Pavasario balsai. Lyrika, Vilnius: Lietuvių literatūros ir tautosakos institutas, 80 . 
Maironis (1992): «Le château de Trakai», tr. Ugnė Karvelis, en Ugnè Karvelis (ed.) Poésie 92. Revue bimestrielle de la poésie d'aujourd'bui, Paris: La Maison de la Poésie, 85.

Mekas, Jonas (2007): Mano naktys, Vilnius: Baltos lankos.

Mekas, Jonas (2007): Ma vie nocturne, Vilnius: Baltos lankos.

Meras, Icchokas (1995): «Kartus rūgštynių skonis», Apverstas pasaulis, Chicago: Algimanto Mackaus Knygu Leidimo Fondas, 66-8I.

Meras, Icchokas (2003): «Le goût amer de l'oseille», tr. Marielle Vitureau, en Loreta Macianskaite (ed.) Des âmes dans le brouillard, Caen: Presses Universitaires de Caen, 85-103.

SAvickis, Jurgis (I997): «Fleita». Vasaros kaitros, Vilnius: Baltos lankos, 36-44.

SAvickis, Jurgis (200I): «La flûte», tr. Marielle Vitureau, Cabiers lituaniens, nr. 2, 50-57.

Savickis, Jurgis (2006): «Raudoni batukai». Noveles, Vilnius: Z̆altvykslè, 52-62.

SAvickis, Jurgis (2003): "Les souliers rouges», tr. Marielle Vitureau, en Loreta Macianskaite (ed.) Des âmes dans le brouillard, Caen: Presses Universitaires de Caen, 239-256.

ŽIndŽIUVIENĖ-DensNYTĖ, Elena (2000): «Baltasis bokštas», Žiobiškis, Vilnius: Versmè, 538-588.

ŽIndŽIuvienĖ-DeksNyté, Elena (2008): «La tour blanche», tr. Jean-Claude Lefebvre, Cabiers lituaniens, 9, 49-54.

\section{Bibliographie Secondaire}

Cordonnier, Jean-Louis (1995): Traduction et culture, Paris: Les Éditions Didier.

Durieux, Christine (I998): «La traduction, transfert linguistique ou transfert culturel ?», Revue des lettres et de traduction, 4, I3-29.
Eco, Umberto (2006): Dire presque la même chose. Expériences de traduction, trad. Myriem Bouzaher, Paris: Éditions Grasset \& Fasquelle.

Gadamer, Hans-Georg (I999): Istorija. Menas. Kalba, trad. Arūnas Sverdiolas, Vilnius: Baltos lankos.

IsRAËL, Fortunato (2005): «Pricipes pour une pédagogie raisonnée de la traduction: le modèle interprétatif», en Fortunato IsRaËL, Marianne LEDERER (ed.), La Théorie Interprétative de la Traduction III (de la formation à la pratique professionnelle), ParisCaen: Lettres modernes minard, 6I-73.

Karvelis, Ugnè (I998): La littérature lituanienne, Strasbourg: Association Alsace-Lituanie.

Kristeva, Julia (i969): "Le mot, le dialogue et le roman», Sèméiotikè, Paris: Seuil.

Lederer, Marianne (1994): La traduction aujourd'bui. Le modèle interprétatif, Paris: Hachette.

Lefebvre, Jean-Claude (2005): «Regards sur la Lituanie: Lokis de Mérimée», Cabiers lituaniens, 6, 23-28.

LeonavičıenĖ, Aurelija (2013): «Lietuvių literatūros kūrinių intertekstinių reikšmių interpretacija ir vertimas ì prancūzų kalbą», Respectus philologicus, $23(28), 97$-IO8.

LeonavičIené, Aurelija (20I4): Kultūriniu teksto reikšmiu interpretacija ir vertimas, Kaunas: Technologija.

RAGAIŠIENĖ, Irena (2008): «Intertekstualumo ir kultūrinès informacijos perteikimo problemos Helenos Fielding Bridžitos Džouns dienoraščio vertime ì lietuvių kalbą", Kalbu studijos / Studies about languages, I3, 44-50.

Ricoeur, Paul (2004): Sur la traduction, Paris: Bayard.

Ricoeur, Paul (2010): Apie vertimq, trad. Paulius Garbačiauskas, Vilnius: Aidai.

Roux-FAuCARD, Geneviève (2006): «Intertextualité et traduction», Meta, 5I(I), 98-II8.

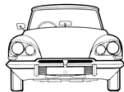

231 